Hydraulic Engineering Repository

Ein Service der Bundesanstalt für Wasserbau

Scannel, Joseph P.; Baribault, Marc K.

Georgia DOT's Implementation of BridgeWatch

Verfügbar unter / Available at:

https://hdl.handle.net/20.500.11970/100223

Vorgeschlagene Zitierweise / Suggested citation:

Scannel, Joseph P.; Baribault, Marc K. (2010): Georgia DOT's Implementation of BridgeWatch. In: Burns, Susan E.; Bhatia, Shobha K.; Avila, Catherine M. C.; Hunt, Beatrice E. (Hg.): Proceedings 5th International Conference on Scour and Erosion (ICSE-5), November 7-10, 2010, San Francisco, USA. Reston, Va.: American Society of Civil Engineers. S. 924-930. 


\title{
Georgia DOT's Implementation of BridgeWatch ${ }^{\circledR}$
}

\author{
Joseph P. Scannell ${ }^{1}$, President and Marc K. Baribault ${ }^{2}$, Meteorologist
}

\begin{abstract}
${ }^{1}$ USEngineering Solutions Corporation, 179 Allyn Street, Hartford, CT 06103; PH (860) 524-9110; email: jscannell@usengineeringsolutions.com

${ }^{2}$ USEngineering Solutions Corporation, 179 Allyn Street, Hartford, CT 06103; PH (860) 524-9110; email: mbaribault@usengineerignsolutions.com
\end{abstract}

\begin{abstract}
Transportation professionals are charged with the formidable task of protecting, maintaining, and replacing bridges in a cost-effective manner to allow safe passage by over 230 million road and rail vehicles daily and commercial vehicles transporting over $\$ 8.4$ trillion of cargo annually. A bridge failure is costly in terms of life and property. However, many failures can be prevented, delayed, or mitigated through monitoring, early warning, and well-coordinated emergency response protocols.

During the fall of 2008, the Georgia Department of Transportation ("GDOT") was looking for a way to monitor their scour-critical bridge population and incorporate this effort into the development of plans-of-action ("POA") for seventysix (76) scour-critical bridges. The monitoring system they chose is a web-based software system known as BridgeWatch ${ }^{\circledR}$. The system utilizes patented technology that collects and processes real-time data to provide automated early warning to engineers of any potentially-destructive environmental conditions in and around their structures so they can effectively respond to protect life and property.

GDOT engineers were given the ability to populate their BridgeWatch system with inventory data, personnel contact information, and structure specific thresholds, to monitor for environmental conditions such as accumulated precipitation, increased river flows, hurricane induced tidal surge, and seismic events. The system enables owners and operators to make informed decisions about public safety, in real-time, and effectively dispatch emergency personnel, safety inspectors and maintenance workers, prior to flooding or other destructive environmental events which adversely affect bridges.
\end{abstract}




\section{SYSTEM DESCRIPTION}

The GDOT-version of BridgeWatch is a web-based software system designed to access real-time meteorologic, hydrologic, seismologic data sources, analyze site specific data, and evaluate said data against established thresholds pertaining to specific-related structures to determine if, when, and how a user of the system should be alerted. The system enables more effective communication by automatically contacting key personnel and informing them of a potentially destructive event, so they can respond with an appropriate POA protocol. BridgeWatch's capabilities allow for the early detection of potential hazards and allow for the GDOT to initiate their emergency response actions. Through an automated notification process, users receive system-generated watch lists of structures experiencing critical flooding events. A watch list, which can be sorted for specific attributes, establishes a priority list of structures requiring inspections or other emergency actions.

\section{EXTERNAL DATA SOURCES}

BridgeWatch accesses several external data sources in real-time, including, but not limited to: data derived by the National Oceanographic Atmospheric Administration ("NOAA"), the United States Geological Survey ("USGS"), and the National Weather Service ("NWS"). The NWS makes available warnings that enable the system to provide early notification for flooding activity. The system gathers and processes relevant river data collected by USGS real-time stream flow stations.

NOAA publishes real-time Next Generation Radar ("NEXRAD") rainfall accumulation data sets for processing by the system algorithms. GDOT's version of BridgeWatch has been configured to interact with the above external data sources. The system can be further configured to include other data sources such as, bridge specific monitoring devices, seismic activities, and other related asset location and disposition information.

\section{NWS Data Source}

The BridgeWatch system notifies users when the NWS flood and flashflood warnings ("FLW" and "FFW," respectively) are issued and processed. These warnings may or may not require action by the user depending on GDOT's existing protocols. FLW and FFW data are obtained from the Interactive Weather Information Network ("IWIN") web interfaces. The warning data is formatted as Advanced Weather Interactive Processing System ("AWIPS") text data sets as identified in the AWIPS header. Referring to Figure 1, the heading (shaded in yellow), includes an abbreviated World Meteorological Organization ("WMO") code which identify all types of weather data. Imbedded in this weather data are bulletins that represent FLW and FFWs (shaded in red) that are parsed by the system's data handler. Located at the bottom of each warning is a series of four-digit numbers that identify the location of the warning area shaded in green. 


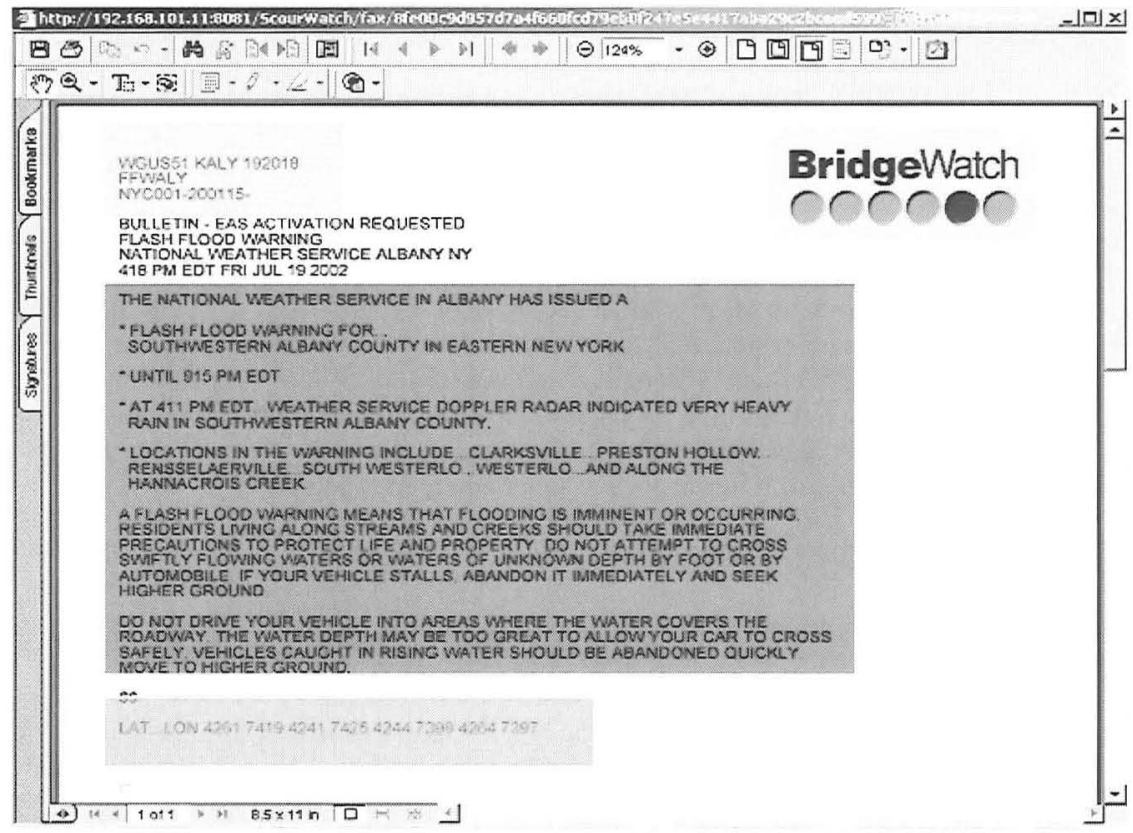

Figure 1. A NWS Flashflood Warning captured and processed by BridgeWatch

\section{USGS Data Source}

BridgeWatch accesses USGS' real-time river data from Georgia's gage network at regular intervals. The system gathers and decodes data from real-time stream flow stations for comparison against a static Event Threshold Database ("ETD") as provided by GDOT. The ETD contains thresholds for the 2, 5, 10, 25, 50, 100,200 and 500-year peak discharge flows for each real-time gage the system interrogates. The system will continually compare data and generate further calculations when one of the thresholds is reached or exceeded. When a threshold is exceeded, the system applies the threshold value to the corresponding bridge associated with that particular gage. GDOT has established the criteria for the bridge associations to a particular gage when the bridge is common to a particular watershed. However, due to the fact that not all watersheds contain gages, not all structures will have associations to gages. The system will generate an event once it has processed the gauged bridge associations and threshold criteria. 


\section{NOAA Data Source}

The system accesses NEXRAD imagery data sets provided by NOAA in order to perform calculations for threshold comparisons and for direct interface display. GDOT has provided values for these thresholds for each bridge in the system according to six major storm intensities, termed the 2, 5, 10, 25, 50 and 100-year return frequency rainfall intensities. Like the ETD for the stream flows, BridgeWatch will use the rainfall ETD to establish the criteria for creating watch lists. During these rainfall events, NEXRAD binary radar imagery is obtained and decoded by the system data handler. NEXRAD data sets are updated approximately every 5 to 10 minutes, depending on the radar's configuration. However, the most useful data sets are the One-Hour, Three-Hour and Storm Total Precipitation accumulation products. These data sets contain raster imagery identifying accumulated rainfall for the coverage area of each NEXRAD installation. Once a NEXRAD rainfall threshold is exceeded, the system data handler then creates a corresponding watch list of bridges. Threshold exceedance is calculated using the NEXRAD overlay and a bridge's basin of influence.

\section{INTERNAL DATA SOURCES}

The system houses several internal data sources as separate databases within the central database: the GDOT BridgeWatch system consists of five primary databases. The first three consist of data provided by the GDOT Bridge Maintenance Unit ("BMU"): profiles, bridges, and threshold databases. The fourth database, gages, consists of a list of real-time gages that are interrogated by the system. The fifth database, tracking hydrologic events, is generated and updated through the use of the application which archives active events.

\section{Profiles and Subscriptions}

GDOT user information is maintained in the system's profiles database. Each GDOT has a unique profile containing username, product subscriptions, contact information (cell phone, pager, fax and email) and a geographic area of responsibility. The GDOT BridgeWatch system has over a hundred such profiles, or users, ranging from field staff to GDOT BMU managers. Each profile has a unique set of subscriptions resident the system. The BridgeWatch subscription processor crossreferences the profile database to properly disseminate alert notifications as real-time event information is processed and products are generated. User profiles are maintained and updated quarterly by the GDOT BMU.

\section{Bridges and Threshold Databases}

The GDOT BridgeWatch database includes seventy-six (76) scour-critical bridges throughout Georgia. This database contains bridge specific information extracted from the reporting items in Georgia's bridge information management system in addition to photos, reports, plans, and digital POAs. Bridge number, carried and crossed features, and item 113 codes are just a few of the many data fields 
housed within the system. The system displays bridge data from the bridge database to the user via the web interface.

The thresholds database, generated from data provided by GDOT, contains the exceedance values for rainfall intensity (measured in inches) and stream flow (measured in cubic feet per second) relating to those bridges in the bridge database. The GDOT BridgeWatch data handler is constantly checking thresholds against realtime data sources. Once a threshold exceedance is discovered, the data handler packages the information as a product to be processed by the subscription processor which translates products into alert notifications.

\section{Events and Alert Notifications}

The events database is generated and maintained by the GDOT BridgeWatch system; it records every alert notification generated by the system and maintains an events archive. This archive is useful in reviewing past events and assembling system reports. BridgeWatch displays event data from the events database to the user via the system's administrative panel. Since inception, the GDOT system has identified more than thirty rainfall events and more than a dozen river flow events as associated to Georgia's scour-critical bridge population. Alert bridges will appear on the geospatial environment of the system interface as red icons (Figure 2). Users can click on these icons to learn more about the alert type and review pertinent bridge information. GDOT field engineers can access the system remotely enabling them to publish on-site information in real-time via the system's ticket feature. GDOT staff use tickets to document their response to alert notifications.

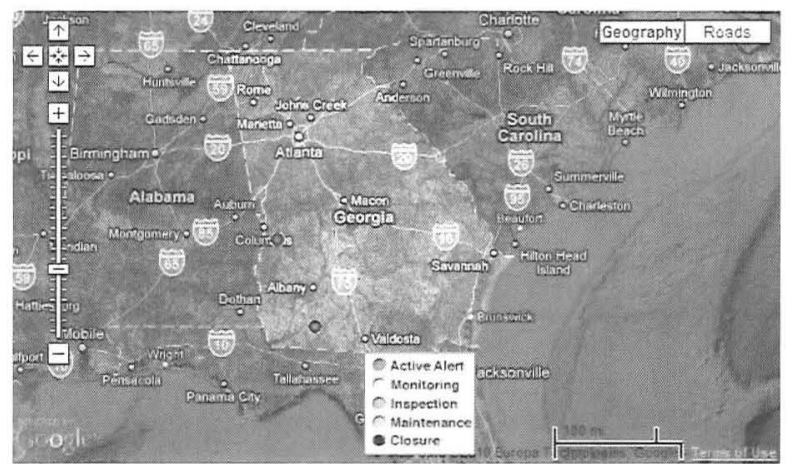

Figure 2. Active Alert Bridges displayed as red icons 


\section{PLANS OF ACTION}

Like all states, Georgia was required to develop POAs in accordance with an FHWA federal mandate related to scour-critical bridges. However, Georgia has uniquely developed, integrated, and maintained POAs for all 76 scour critical bridges using the BridgeWatch system. The GDOT digital POA has four main tabs (Bridge Data, Monitoring Plan, Hydraulics Data, and Comments). The Bridge Data tab can be seen in Figure 3 below.

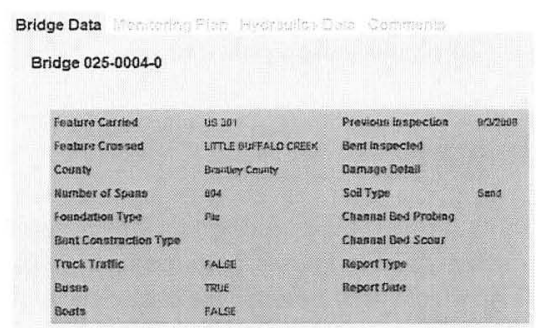

Figure 3. Georgia Digital POA's Bridge Data

The Monitoring Plan consists of on and off-site monitoring expectations for each scour-critical bridge. The GDOT system provides the users with the ability to leverage the benefits of off-site monitoring by using the real-time data aggregation capabilities of the system. Many situations can be monitored remotely prior to sending inspection teams to a scour critical bridge site for on-site monitoring. Off-site monitoring is a cost-effective way to only send inspection teams when and where their services are going to be valuable. Figure 4 illustrates Georgia's monitoring plan.

Monitoring Plan

Bridge 025-0004-0

Othsite Morktaning:

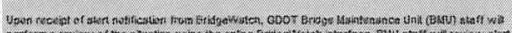

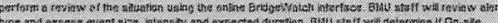

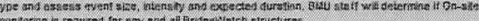

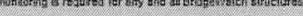

On-sien Nonitoringe

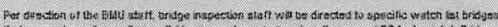

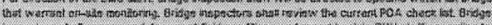

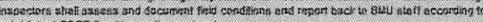
eatiblshed GoOT Abed inspection pretacet.

Figure 4. Georgia Digital POA's on and off-site monitoring data 


\section{CURRENT UPDATES}

Over the past several years, GDOT has become more aware of the capabilities of this population-based approach to monitoring scour-critical bridge populations and have decided to further invest in adding probabilistic storm surge ("P-Surge") data to their monitoring data sources. This will enable GDOT officials to establish thresholds and be alerted when coastline areas are expected to experience coastal inundation from hurricanes making landfall or influencing the eastern coastline of Georgia. GDOT officials will use this data set for the ability to properly allocate assets during and after major hurricanes impact their state. As of the date of this publication, GDOT was still in the implementation phase of this product and therefore no images were available.

\section{CLOSING STATEMENT}

The BridgeWatch web-based solution uniquely aggregates public and private data sources that, until now, have been maintained and published independent of one another. The GDOT BridgeWatch system has created a synthesis of these data sources to the extent they are complimentary in providing a comprehensive real-time understanding of the environmental hazards affecting the safe operation of state owned bridges. The GDOT BridgeWatch system has been employed for two years. During this time GDOT bridge maintenance engineers have been notified and responded to more than thirty (30) system-generated alerts which have identified locations where bridges needed to be repaired and even closed due to flood related events.

The employment of new monitoring technology will be the only cost-effective way to increase safety levels and make more timely informed decisions regarding the management of public infrastructure: public safety through real-time structure monitoring. 